\title{
Involved-Field Radiation Therapy
}

National Cancer Institute

\section{Source}

National Cancer Institute. Involved-Field Radiation Therapy. NCI Thesaurus. Code C113812.

Radiation treatment delivered only to the areas of the body that are affected, used primarily in the treatment of lymphoma. 